\title{
CONTROLE DE Cyperus difformis L. POR HERBICIDAS ALS E MIMETIZADORES DE AUXINAS
}

\author{
CONTROL OF Cyperus difformis BY ALS HERBICIDES AND AUXIN MIMICS
}

Mariane Peripolli ${ }^{\mathrm{a} *}$, Sylvio Henrique Bidel Dornelles ${ }^{\mathrm{b}}$, Vinicius Severo Trivisiol ${ }^{\mathrm{a}}$, Elisandro Azeredo Nunes ${ }^{\mathrm{b}}, \mathrm{Jéssica} \mathrm{Cezar} \mathrm{Cassol}^{\mathrm{b}}$, Aliana Teixeira Flores $^{\mathrm{c}}$

${ }^{a}$ Departamento de Fitotecnia, Universidade Federal de Santa Maria, Rio Grande do Sul, Brasil. ${ }^{\text {b}}$ Departamento de Biologia, Universidade Federal de Santa Maria, Rio Grande do Sul, Brasil. 'Colaboradora externa, Biomonte Pesquisa e Desenvolvimento, Rio Grande do Sul, Brasil.

*Autor correspondente: mperipolli@gmail.com.

\section{INFORMAÇÕES DO ARTIGO}

\section{Histórico do artigo:}

Recebido: 21 Outubro 2019.

Aceito: 16 Setembro 2020.

Publicado: 02 Outubro 2020.

\section{Palavras-chave/Keywords:}

Arroz/ Rice.

Biótipos/ Biotypes.

Controle químico/ Chemical Control.

Resistência/ Resistance.

Direito Autoral: Este é um artigo de acesso aberto distribuído sob os termos da Licença Creative Commons, que permite uso, distribuição e reprodução irrestritos em qualquer meio, desde que $\mathrm{o}$ autor $\mathrm{e}$ a fonte originais sejam creditados

\section{Citação deste artigo:}

PERIPOLLI, M.; DORNELLES, S. H. B.; TRIVISIOL, V. S.; NUNES, E. A.; CASSOL, J. C.; FLORES, A. T. Controle de Cyperus difformis 1. por herbicidas als e mimetizadores de auxinas. Revista Brasileira de Herbicidas, v. 19, n. 2. 2020.

\begin{abstract}
RESUMO
A ocorrência de biótipos de Cyperus difformis L. com característica de resistência à aplicação de herbicidas no setor da orizicultura é uma preocupação mundial. Nesse sentido, objetivou-se avaliar a eficiência do controle de $C$. difformis por herbicidas inibidores da acetolactato sintase (ALS) e mimetizador de auxina. O delineamento experimental foi inteiramente casualizado, com quatro repetições, totalizando 252 unidades experimentais compostas por vasos com 12 quilogramas de solo e os tratamentos arranjados em esquema fatorial $3 \times 7 \times 2 \times 2$ : três herbicidas (dois inibidores da ALS pirazossulfurom-etílico e bispiribaque-sódico e um mimetizador de auxina triclopir-butotílico); sete doses dos herbicidas $(0.0 \mathrm{x}, 0.5 \mathrm{x}, 1 \mathrm{x}, 2 \mathrm{x}, 4 \mathrm{x}, 8 \mathrm{x}$ e $16 \mathrm{x}$ a dose de registro); dois biótipos de $C$. difformis (Santa Rosa do Sul e Meleiro, Santa Catarina (SC)); e duas umidades de solo $(100 \%$ saturado e lâmina d'água de $5,0 \mathrm{~cm}$ ). O biótipo $C$. difformis de Meleiro-SC é $8 \mathrm{x}$ $\left(320\right.$ g. i.a. ha $\left.{ }^{-1}\right)$ mais resistente a dose de registro do bispiribaque-sódico, já o biótipo de Santa Rosa do Sul-SC é mais sucetivel, reduzindo $50 \%$ da massa seca da parte aérea com $0.5 \mathrm{x}$ da dose de registro $\left(20 \mathrm{~g}\right.$ i.a. ha $\left.^{-1}\right)$. O pirazossulfurom-etílico demostrou-se ineficiente na redução da massa seca da parte aérea em ambos os biótipos. Os biótipo $C$. difformis de Santa Rosa do Sul-SC e Meleiro-SC, nas duas condições de umidade do solo são suscetíveis ao triclopir-butotílico, reduzindo $50 \%$ da massa seca da parte aérea com $0.5 \mathrm{x}$ $\left(166\right.$ g. i.a. ha $\left.^{-1}\right)$ a dose de registro.
\end{abstract}


M. PERIPOLLI et al.

\section{Introdução}

O arroz (Oryza sativa L.) caracteriza-se como a base alimentar de mais de três bilhões de pessoas no mundo (SOSBAI, 2018). É o segundo cereal mais produzido no mundo, chegando a 718 milhões de toneladas, em uma área aproximada de 161,5 milhões de hectares (USDA, 2019). O Rio Grande do Sul é o maior produtor nacional, responsável por $2 / 3$ da produção ( 8,7 milhões de toneladas), em uma área de 1,1 milhão de hectares (CONAB, 2019).

O controle de plantas daninhas no arroz é realizado, quase que exclusivamente, com o manejo químico, principalmente com herbicidas inibidores da enzima acetolactato sintase (ALS), devido sua alta eficiência em baixas doses (PACANOSKI; GLATKOVA, 2009; SOSBAI, 2014; CHIAPINOTTO et al., 2017). Estes herbicidas tornaram-se ferramentas importantes para a agricultura a partir dos anos 80, e passaram a ser utilizado em larga escala. Entretanto, a resistência a inibidores de ALS atinge cerca de $52 \%$ dos casos registrados no mundo (HEAP, 2009).

Cyperus difformis L. é uma planta daninha altamente competitiva com a cultura do arroz irrigado, pois é adaptada à ambientes alagadiços, suportando até pequenas lâminas de água (VALVERDE et al., 2014; TIAN et al., 2020), sendo amplamente disseminada em regiões arrozeiras do mundo todo. No Brasil, é relatada nos estados do Rio Grande do Sul (RS) e Santa Catarina (SC), onde provavelmente sua introdução tenha ocorrido por meio da contaminação junto a sementes de arroz. C. difformis é uma planta anual, metabolismo fotossintético tipo $\mathrm{C}_{3}$, cosmopolita e pouco exigente quanto ao tipo de solo, altamente competitiva, devido à grande quantidade de perfilhos e sementes e rápido crescimento, podendo completar seu ciclo em aproximadamente 60 dias (PEDROSO et al., 2019).

$\mathrm{O}$ controle de Cyperus difformis, no sistema

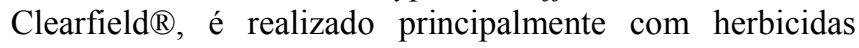
inibidores da enzima ALS. Entretanto, segundo Noldin, Eberhardt e Rampelotti (2002), as plantas de C. difformis apresentam resistência aos herbicidas inibidores da ALS, desde 2000, no Brasil. Em 2018, existem biótipos com resistência a dois mecanismos de ação (inibidores da enzima ALS e inibidor do FSII), dentre estes, no Brasil são registrados casos para mecanismos inibidores da enzima ALS (HEAP, 2017).

A resistência à herbicidas é definida como a capacidade, inerente e herdável, de um biótipo em sobreviver e se reproduzir após exposição ao mesmo (CHRISTOFFOLETI; LÓPEZ OVEJERO, 2008). O uso excessivo de um único herbicida, acaba exercendo pressão de seleção, onde somente plantas resistentes permanecerão na área (BONNY, 2011).

Neste contexto, com o surgimento de biótipos resistentes nas lavouras de arroz irrigado no estado de Santa Catarina (OSUNA et al., 2002; GALON et al., 2008; AGOSTINETTO et al., 2011), objetivou-se com esse estudo avaliar a eficiência do controle de $C$. difformis por herbicidas inibidores da ALS (pirazossulfurom-etílico e bispiribaque-sódico) e um mimetizador de auxina (triclopirbutotílico).

\section{Materiais e Métodos}

O experimento foi realizado em casa de vegetação durante o ano agrícola de 2016/2017. O delineamento experimental utilizado foi o inteiramente casualizado, arranjado em esquema fatorial $3 \times 7 \times 2 \times 2$, com quatro repetições, sendo os fatores: três herbicidas (dois inibidores da ALS (pirazossulfurom-etílico (Sirius $250 \mathrm{SC}, 250 \mathrm{~L}^{-1} \mathrm{~g}$ i.a., SC, Iharabras) e bispiribaque-sódico (Nominee $400 \mathrm{SC}$, $400 \mathrm{~L}^{-1}$ g i.a., SC, Iharabras)) e um mimetizador de auxina (triclopir-butotílico (Triclon, $680 \mathrm{~L}^{-1} \mathrm{~g}$ i.a, CE, UPL) mais $0,25 \% \mathrm{v} / \mathrm{v}$ de adjuvante (Lanzar, $280 \mathrm{~L}^{-1} \mathrm{~g}$ i.a, CE, Arysta); sete doses crescentes dos herbicidas $(0.0 \mathrm{x}, 0.5 \mathrm{x}, 1 \mathrm{x}, 2 \mathrm{x}, 4 \mathrm{x}$, $8 \mathrm{x}$ e 16x a dose de registro; dois biótipos de $C$. difformis (Santa Rosa do Sul e Meleiro-SC); duas umidades de solo ( $100 \%$ de solo saturado e lâmina d'água de 5,0 cm). As 252 unidades experimentais foram compostas por vasos plásticos flexíveis (Nutriplant) de 11 litros de volume, preenchidos com $12 \mathrm{~kg}$ de solo classificado como Argilossolo Vermelho Arênico distrófico. Segundo análise química e física do Laboratório de Física do Solo - UFSM o solo apresentou: $\mathrm{pH}$ em água $(1: 1)=4,25$; matéria orgânica $=2,3 \% ; \mathrm{P}=3,3 \mathrm{mg} \mathrm{dm}^{-3} ; \mathrm{K}=0,119 \mathrm{cmolc} \mathrm{dm}^{-3} ; \mathrm{Ca}=0,3$ cmolc dm ${ }^{-3} ; \mathrm{Mg}=0,1 \mathrm{cmol}_{\mathrm{c}} \mathrm{dm}^{-3} ; \mathrm{H}+\mathrm{Al}=7,3 \mathrm{cmol}_{\mathrm{c}} \mathrm{dm}^{-3}$; CTC efetiva $=2,7 \mathrm{cmol}_{\mathrm{c}} \mathrm{dm}^{-3}$; Saturação Bases $=7 \%$; total areia $=41,228 \mathrm{~kg} \mathrm{~kg}^{-1}$; silte $=41,972 \mathrm{~kg} \mathrm{~kg}^{-1}$; argila: $16,8 \mathrm{~kg}$ $\mathrm{kg}^{-1}$.

A determinação da Capacidade de retenção de água do solo (CRA) foi realizada através da secagem até massa constante (estufa à $70{ }^{\circ} \mathrm{C}$ ). Após, aferiu-se a capacidade de retenção de água do solo. No vaso a ser utilizado contendo uma massa de solo conhecida continha orifícios em sua base para a drenagem do excesso de água. $\mathrm{O}$ vaso foi irrigado até ocorrer a saturação do solo, sendo que, posteriormente, aguardou-se até o momento em que o mesmo atingiu massa constante. Assim, através da diferença de massa do vaso com solo seco para o vaso com solo úmido, obteve-se o valor da quantidade de água para atingir 100\% da capacidade de retenção de água. Para a determinação da condição hídrica do solo (100\% da CRA) foram utilizadas as seguintes fórmulas adaptadas (SCHWAB, 2011):

MV100\% $=($ MVCRA - MVseco $) \times 1,0+$ MVseco

Em que: $M V n \%$ é a massa do vaso para cada um dos tratamentos; MVCRA é a massa do vaso na capacidade de retenção de água; MVseco corresponde a massa do vaso preenchido com solo seco.

O fornecimento das referidas quantidades de água, ocorreu regularmente por meio do método de pesagem, utilizando uma balança eletrônica marca ACS System com precisão de $5 \mathrm{~g}$, sendo adicionado água até atingir a massa total pré-determinada (vaso + solo seco + volume de água para atingir $100 \%$ da CRA do solo) e mantendo a lâmina d'água de $5 \mathrm{~cm}$.

Os biótipos de $C$. difformis foram coletados em duas lavouras comercias de arroz irrigado, com suspeita de resistência de herbicidas inibidores da ALS, nos municípios de Meleiro e Santa Rosa do Sul, Santa Catarina. As plantas, com aproximadamente $2 \mathrm{~cm}$, provenientes dos dois biótipos, foram transplantados para os vasos plásticos, mantendo-se uma planta por recipiente. 
A aplicação dos tratamentos foi realizada quando os biótipos de $C$. difformis encontravam-se no estádio de desenvolvimento de duas a quatro folhas, aproximadamente $10 \mathrm{~cm}$. Utilizou-se um pulverizador costal pressurizado a $\mathrm{CO}_{2}$ comprimido, munido de um bico de jato plano, tipo leque, modelo XR 110.02, com pressão de trabalho de $172.37 \mathrm{kPa}$, barra postada a $50 \mathrm{~cm}$ da superfície do alvo, proporcionando uma taxa de aplicação com volume de 200 $\mathrm{L} \mathrm{ha}^{-1}$.

A avaliação de controle das plantas foi realizada visualmente, aos 3, 7, 14, 21 e 28 dias após aplicação (DAA) dos tratamentos, tempo suficiente para visualização dos sintomas de fitointoxicação nas plantas-alvo. Realizouse análise visual conforme escala da Sociedade Brasileira da Ciência das Plantas Daninhas (SBCPD, 1995) aos 28 DAA. A determinação da massa seca ocorreu aos 28 DAA, tempo suficiente para visualização dos sintomas de fitointoxicação nas plantas-alvo. As plantas foram coletadas, separou-se a parte área a qual foi seca em estufa com circulação forçada de ar, aquecida a $60^{\circ} \mathrm{C}$ até atingir massa constante.

Os dados foram submetidos à aplicação do teste ' $\mathrm{F}$ ' na análise da variância para a verificação das interações entre os fatores, posteriormente, para as interações significativas, os dados foram ajustados ao modelo de regressão não linear do tipo log-logístico (SEEFELDT; JENSEN; FUERST, 1995).

$$
Y=\frac{A}{\left[1+\left(\frac{X}{B}\right)^{C}\right]}
$$

Onde: $\mathrm{Y}=$ controle $(\%)$ ou massa seca $(\mathrm{g})$ aos 20 DAA; $\mathrm{X}=$ dose do herbicida e A, B e C são parâmetros da curva, de modo que $\mathrm{A}=$ diferença entre o ponto máximo e mínimo da curva; $\mathrm{B}=$ dose do herbicida $\left(\mathrm{g} \mathrm{ha}^{-1}\right)$ que corresponde a $50 \%$ do controle ou responsável por reduzir em $50 \%$ o acúmulo de massa da parte aérea seca e $\mathrm{C}=$ parâmetros do modelo que descreve a declividade da curva.

Os parâmetros exigidos na equação foram obtidos através da plotagem dos dados da massa seca da parte aérea (MSPA) de plantas em relação ao tratamento controle. As figuras foram obtidas a partir de uma matriz básica de dados analisada no programa SigmaPlot ${ }^{\circledR}$ versão 11 .

\section{Resultados e Discussão}

Em relação ao fator herbicidas, verificou-se para o bispiribaque-sódico uma interação significativa apenas entre os fatores doses x biótipos, não havendo efeito do fator condições de umidade do solo, assim, os gráficos foram obtidos com a média dos mesmos (Figura 1).

Observa-se uma redução de $50 \%$ da massa seca da parte aérea (MSPA) (g) $\left(\mathrm{ED}_{50}\right)$ do biótipo $C$. difformis de Santa Rosa do Sul-SC, a partir da aplicação do herbicida bispiribaque-sódico na dose de 22,79 g i.a. ha ${ }^{-1}$, sendo a dose necessária para reduzir $50 \%$ a MSPA $(\mathrm{g})$ semelhante à 0,5 dose de registro (20 g i.a. ha $\left.{ }^{-1}\right)$. Esses resultados corroboram com estudo realizado por Spatt et al. (2015), em que a a aplicação de bispiribaque-sódico foi eficiente no controle, obtendo controle maior que $80 \%$ da espécie em nível de campo (Figura 1).

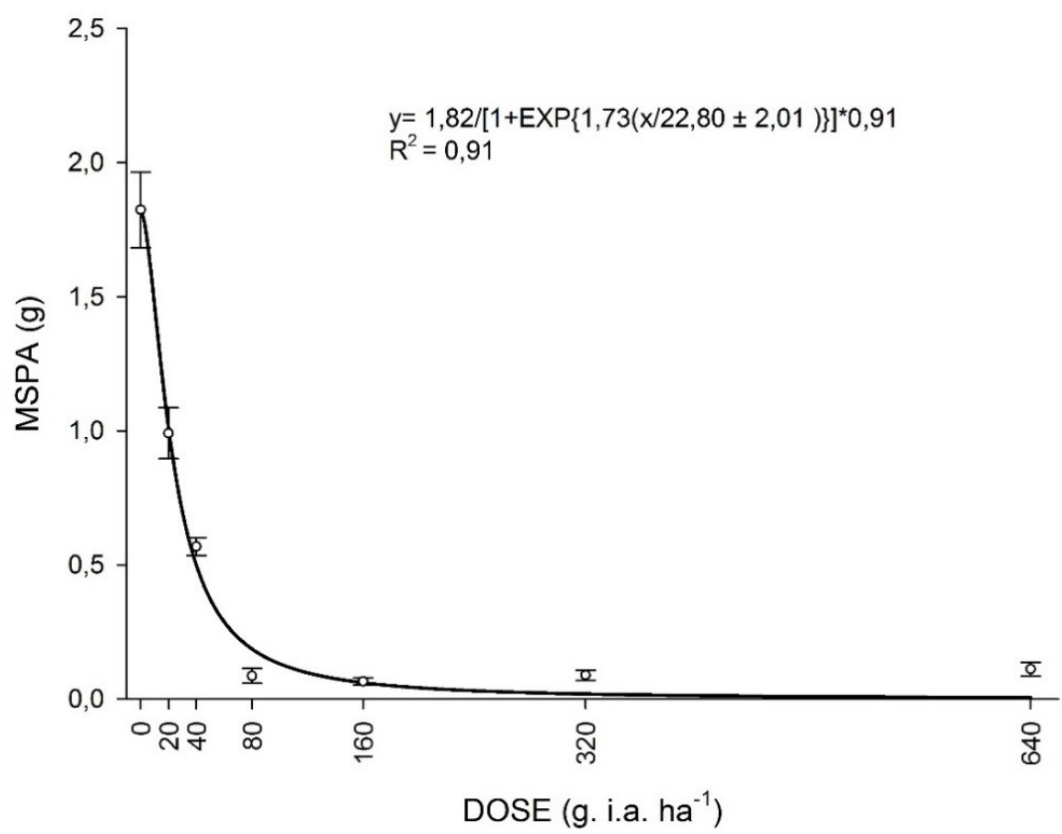

Figura 1. Massa seca da parte aérea (MSPA) (g) do biótipo C. difformis de Santa Rosa do Sul-SC, sob a média das condições de umidade do solo (100\% saturado e lâmina d'água), em função da aplicação pós-emergente do herbicida bispiribaque-sódico nas doses 0.0x (zero); 0.5 x $\left(20\right.$ g. ia. ha $\left.{ }^{-1}\right) ; 1$ x $\left(40\right.$ g. ia. ha ${ }^{-1)} ; 2$ x $\left(80\right.$ g. ia. ha $\left.{ }^{-1}\right) ; 4$ x $\left(160\right.$ g. ia. ha $\left.{ }^{-1}\right) ; 8$ x $\left(320\right.$ g. ia. ha $\left.{ }^{-1}\right)$ e $16 x\left(640\right.$ g. ia. ha $\left.{ }^{-1}\right)$ de registro. Santa Maria-RS, 2017. 
M. PERIPOLLI et al.

A MSPA (g) do biótipo C. difformis de Meleiro-SC somente foi reduzida em $50 \%\left(\mathrm{ED}_{50}\right)$ com a aplição do herbicida bispiribaque-sódico na dose de 322,58 g i.a. ha ${ }^{-1}$, havendo uma resistência do biótipo à aplicação de pelo menos 8 x (320 g. i.a. ha $\left.{ }^{-1}\right)$ a dose de registro para promover uma redução $50 \%$ da MSPA (g) (Figura 2).

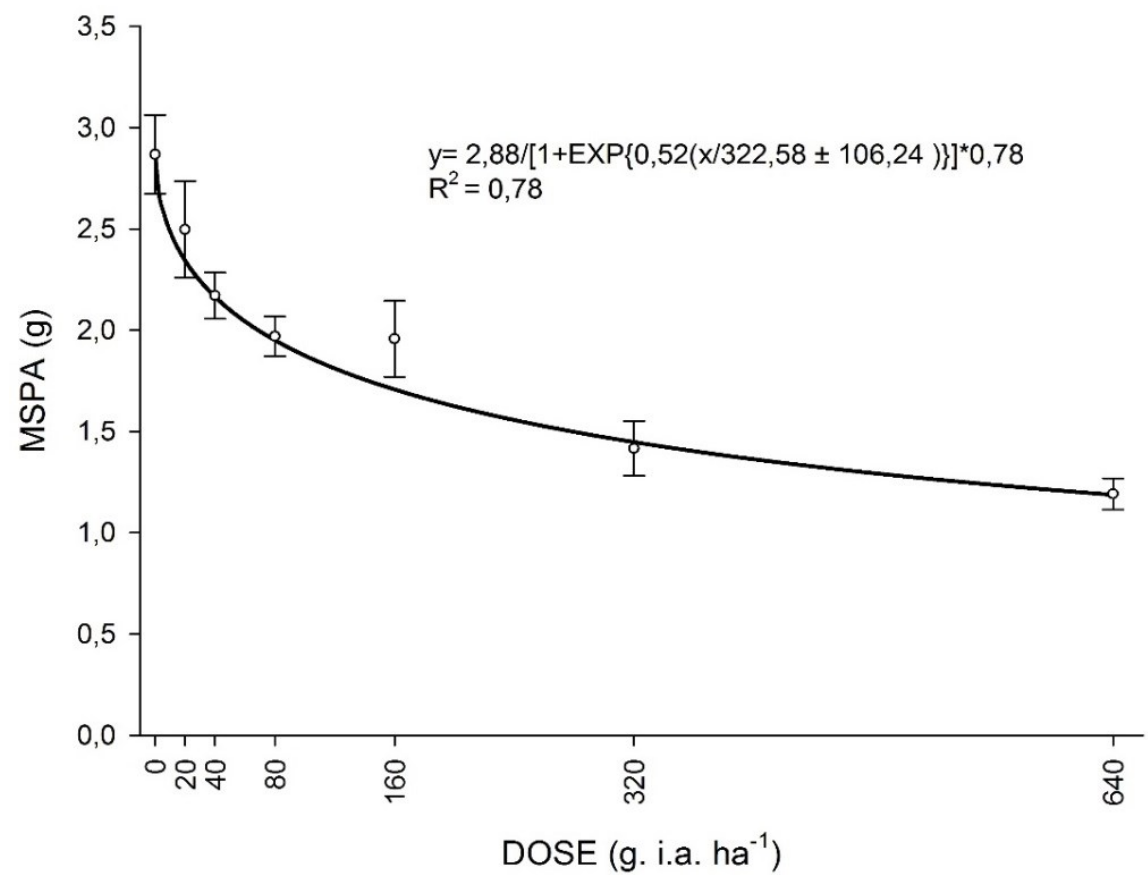

Figura 2. Massa seca da parte aérea (MSPA) (g) do biótipo C. difformis de Meleiro-SC, sob a média das condições de umidade do solo ( $100 \%$ saturado e lâmina d'água), em função da aplicação pós-emergente do herbicida bispiribaque-sódico nas doses $0.0 x$ (zero); 0.5x $\left(20\right.$ g. ia. ha $\left.{ }^{-1}\right) ; 1$ x $\left(40\right.$ g. ia. ha ${ }^{-1)} ; 2 x\left(80\right.$ g. ia. ha ${ }^{-1)} ; 4 x\left(160\right.$ g. ia. ha $\left.{ }^{-1}\right) ; 8 x\left(320\right.$ g. ia. ha $\left.{ }^{-1}\right)$ e 16x $\left(640\right.$ g. ia. ha $\left.{ }^{-1}\right)$ de registro. Santa Maria/ RS, 2017.

Em comparação aos dois biótipos provenientes de Santa Rosa do Sul-SC e Meleiro-SC, verificam-se níveis distintos da redução de MSPA (g) de C. difformis em função da aplicação de bispiribaque-sódico, destacando-se que o biótipo obtido de Meleiro-SC que necessitou de $8 \mathrm{x}$ a dose de registro (320 g. i.a. ha ${ }^{-1}$ ) para promover uma redução de $50 \%$ da MSPA (Figura 2), enquanto que para o biótipo de Santa Rosa do Sul-RS, obteve-se uma redução de $50 \%$ MSPA com $0.5 \mathrm{x}$ a dose de registro (20 g. i.a. ha ${ }^{-1}$ ) (Figura 1). Segundo Ulguim et al. (2019), o gênero Cyperus vem se tornando um problema no que diz respeito a resistência a estes herbicidas, devido ao aumento nas aplicações dos herbicidas ALS nas culturas orizícolas. Assim, podendo ser um dos fatos dos biótipos de Meleiro apresentarem maior resistência devido ao aumento no uso deste grupo químico de herbicidas. Características como uso contínuo, alta eficácia, atividade residual no solo, alta adaptabilidade ecológica do biótipo resistente e mutações pontuais podem conferir resistência de uma planta daninha a um ou mais dos herbicidas inibidores de ALS (CHIAPINOTTO et al., 2017).

Para o herbicida pirazossulfurom-etílico houve uma interação significativa apenas do fator doses, não havendo efeito dos biótipos e condições de umidade do solo, assim, os gráficos foram obtidos com a média dos dois biótipos e das duas condições de umidade do solo (Figura 3).

Os biótipos de $C$. difformis apresentaram um alto nível de insensibilidade ao herbicida pirazossulfurom-etílico (Figura 3). Visto que, o $\mathrm{ED}_{50}$ calculado pela equação extrapolou os valores máximos testados, sendo necessário uma dose superior à $600 \mathrm{~g}$ i.a. ha ${ }^{-1}$ para promover uma redução de 50\% da MSPA (g). Este estudo corrobora com Agostinetto et al. (2011), onde foi necessário utilizar 64x a dose comercial do produto para haver controle das plantas de $C$. difformis, evidenciando assim a resistência da espécie ao herbicida.

Em relação ao herbicida triclopir-butotílico verificou -se uma interação significativa entre todos os fatores (doses x biótipos x condição de umidade do solo) (Figuras 4, 5, 6 e 7). Houve uma redução de $50 \%$ da MSPA (g) do biótipos $C$. difformis de Santa Rosa do Sul-SC na condição de umidade do solo de $100 \%$ saturado, a partir da dose de 100,7 g i.a. ha ${ }^{-1}$ de triclopir-butotílico, sendo inferior à $0,5 \mathrm{x}$ (166 g. i.a. ha ${ }^{-1}$ ) da dose de registro comercialmente (Figura 4). Já para o biótipo, C. difformis de Santa Rosa do Sul-SC, sob condição de umidade do solo de lâmina d'água, verificou-se, uma redução de $50 \%$ da MSPA (g) com a aplicação da dose a partir de 109,89 g i.a. ha-1 correspondendo à 0,5 dose de registro (166 g. i.a. ha $\left.{ }^{-1}\right)$ (Figura 5). Os resultados obtidos de MSPA (g) dos biótipos de $C$. difformis em função da aplicação do triclopirbutotílico nas doses e condições de umidade do solo testadas, evidenciam uma alta suscetibilidade para o triclopir-butotílico. Em estudo realizado por Hofstra, Champior e Dudgale (2006), também foi observado fácil controle da espécie com o uso deste herbicida. 
M. PERIPOLLI et al.

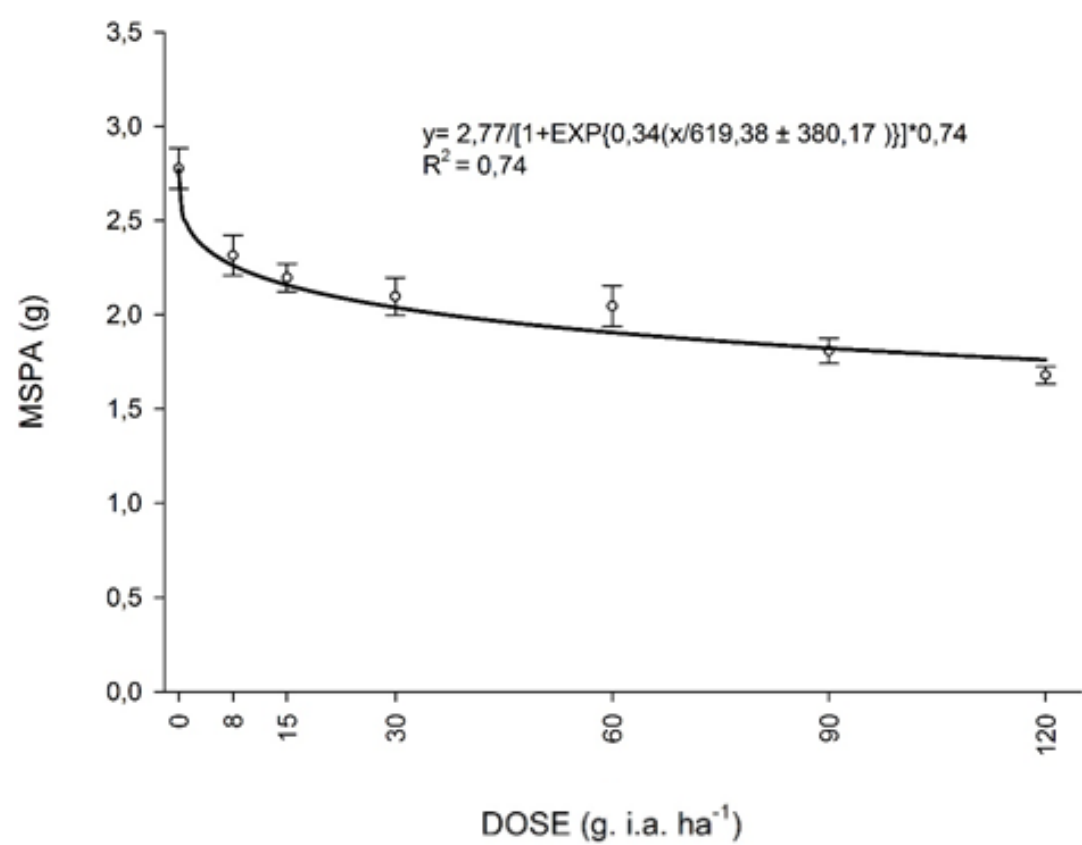

Figura 3. Média da massa seca da parte aérea (MSPA) (g) dos biótipos C. difformis provenientes de Meleiro-SC e Santa Rosa do Sul-SC e condições de umidade do solo (100\% saturado e lâmina d'água), em função da aplicação pós-emergente do herbicida pirazossulfurometílico nas doses $0.0 \mathrm{x}$ (zero); $0.5 \mathrm{x}\left(8 \mathrm{~g}\right.$. ia. ha $\left.{ }^{-1}\right) ; 1 \mathrm{x}\left(15\right.$ g. ia. ha ${ }^{-1)} ; 2 \mathrm{x}\left(30\right.$ g. ia. ha $\left.{ }^{-1}\right) ; \mathrm{x}\left(60\right.$ g. ia. ha $\left.{ }^{-1}\right) ; 8 \mathrm{x}\left(90 \mathrm{~g}^{-}\right.$ia. ha $\left.{ }^{-1}\right)$ e $16 \mathrm{x}$ $\left(120\right.$ g. ia. ha $\left.{ }^{-1}\right)$ de registro. Santa Maria-RS, 2017.

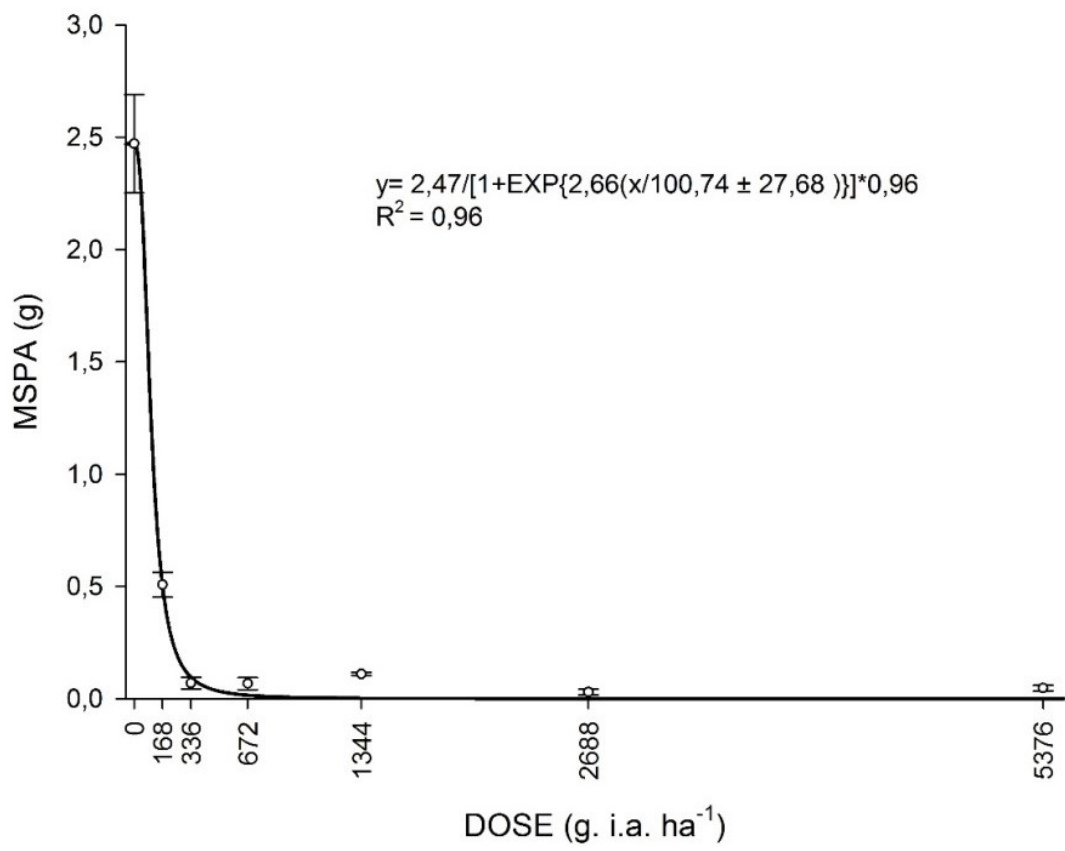

Figura 4. Massa seca da parte aérea (MSPA) (g) do biótipos C. difformis de Santa Rosa do Sul-SC, na condição de umidade do solo de $100 \%$ saturado, em função da aplicação pós-emergente do herbicida triclopir-butotílico nas doses 0.0x (zero); $0.5 \mathrm{x}\left(166 \mathrm{~g}\right.$. ia. ha $\left.{ }^{-1}\right) ; 1 \mathrm{x}(336$ g. ia. ha ${ }^{-1)} ; 2$ x $\left(672\right.$ g. ia. ha ${ }^{-1)} ;$ x (1.344 g. ia. ha $\left.{ }^{-1}\right) ; 8$ x $\left(2.688\right.$ g. ia. ha $\left.{ }^{-1}\right)$ e 16x $\left(5.376\right.$ g. ia. ha $\left.{ }^{-1}\right)$ de registro. Santa Maria/RS, 2017. 
M. PERIPOLLI et al.

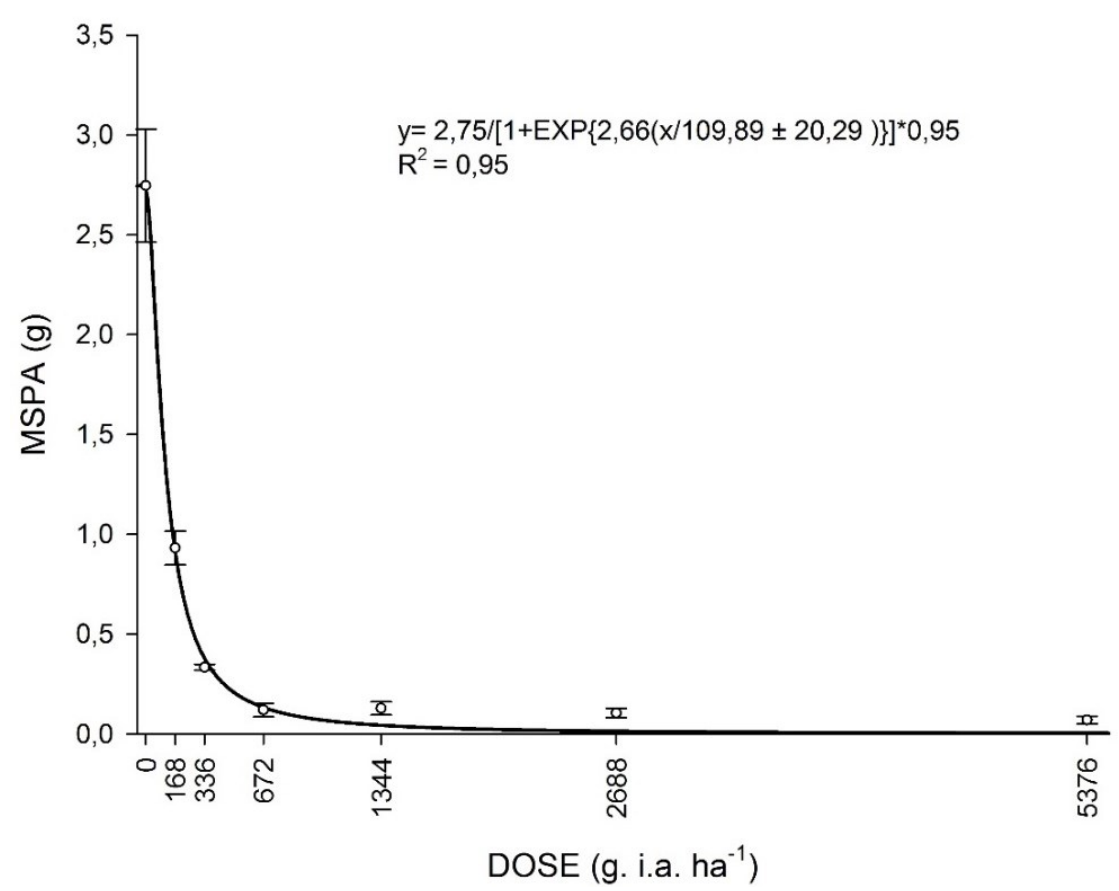

Figura 5. Massa seca da parte aérea (MSPA) (g) do biótipos $C$. difformis de Santa Rosa do Sul-SC, na condição de umidade do solo de lâmina d'água, em função da aplicação pós-emergente do herbicida triclopir-butotílico nas doses $0.0 \mathrm{x}$ (zero); $0.5 \mathrm{x}\left(166 \mathrm{~g}\right.$. ia. ha $\left.{ }^{-1}\right)$; $1 \mathrm{x}(336$ g. ia. ha ${ }^{-1)} ; 2 x\left(672\right.$ g. ia. ha ${ }^{-1)} ; 4 x\left(1.344\right.$ g. ia. ha $\left.{ }^{-1}\right) ; 8$ x $\left(2.688\right.$ g. ia. ha $\left.{ }^{-1}\right)$ e 16x $\left(5.376\right.$ g. ia. ha $\left.{ }^{-1}\right)$ de registro. Santa Maria/RS, 2017.

No que diz respeito ao biótipo $C$. difformis de Meleiro-SC, submetidos a condição de umidade do solo de $100 \%$ saturado, para o herbicida triclopir-butotílico, foi necessária a dose de $89,85 \mathrm{~g}$ i.a. ha ${ }^{-1}$ para promover a redução de 50\% da MSPA (g) o que correspondente a cerca de $25 \%$ da dose de registro comercialmente (Figura 6). Já na condição de umidade do solo de lâmina d'água foi necessária uma dose de 75,15 g i.a. ha ${ }^{-1}$ para reduzir em
$50 \%$ a MSPA (g), correspondendo aproximadamente a $25 \%$ da dose comercial de registro (Figura 7). Não há trabalhos científicos utilizando apenas tricolopir no controle de Cyperus spp., entretanto, Tembe (2014), observou que a utilização de herbicidas hormonais, apresentou uma grande redução na população desta planta daninha aos 30 dias após aplicação.

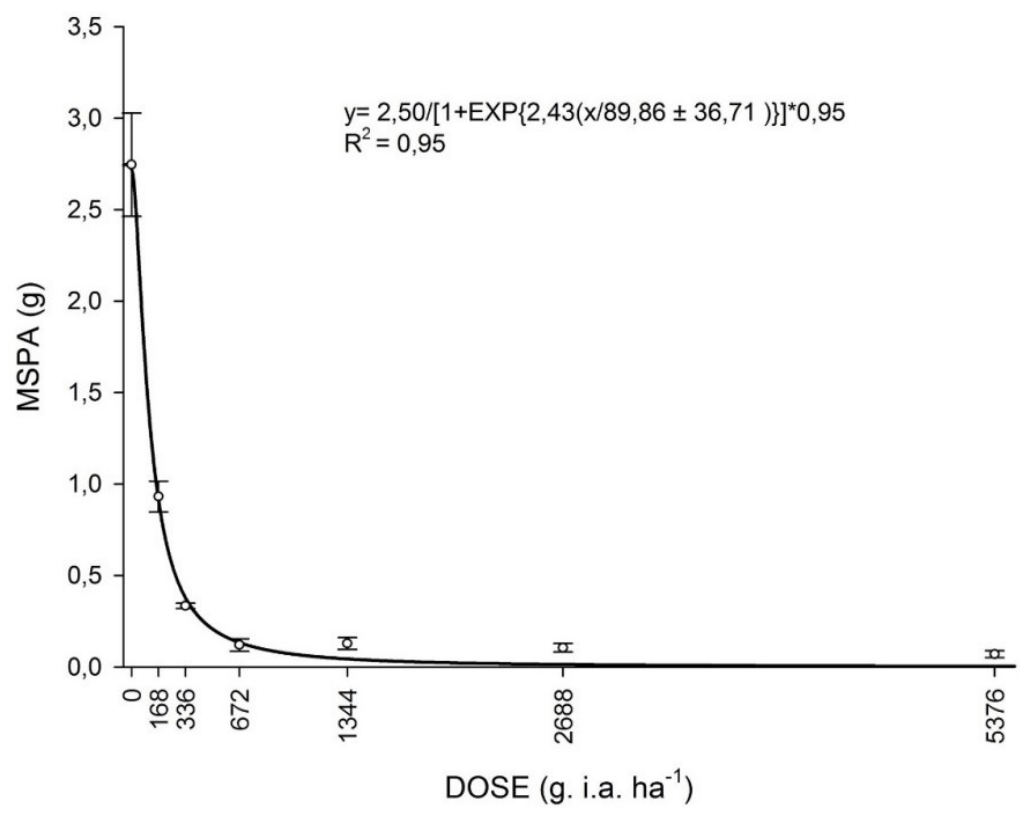

Figura 6. Massa seca da parte aérea (MSPA) (g) do biótipos C. difformis de Meleiro-SC, na condição de umidade do solo $100 \%$ saturado, em função da aplicação pós-emergente do herbicida triclopir-butotílico nas doses $0.0 x$ (zero); 0.5x (166 g. ia. ha-1); 1x (336 g. ia. ha-1); 2x (672 g. ia. ha-1); 4x (1.344 g. ia. ha-1); 8x (2.688 g. ia. ha-1) e 16x (5.376 g. ia. ha-1) de registro. Santa Maria/RS, 2017. 
M. PERIPOLLI et al.

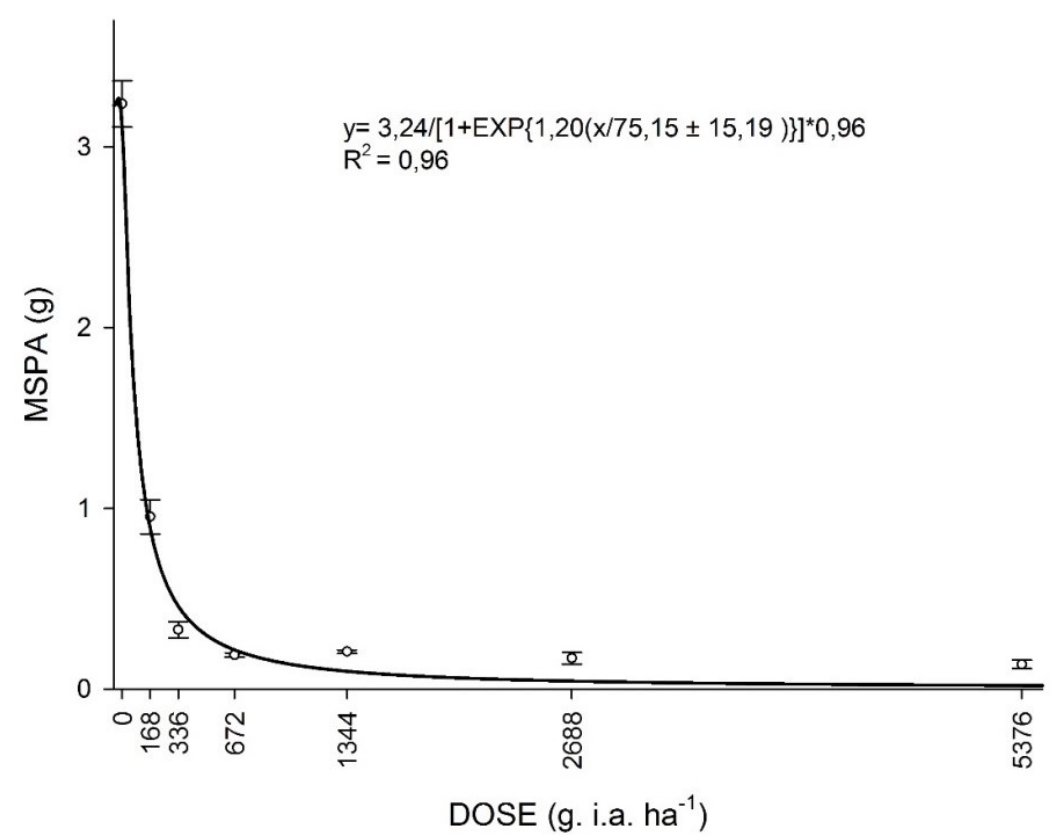

Figura 7. Massa seca da parte aérea (MSPA) (g) do biótipos C. difformis de Meleiro-SC, na condição de umidade do solo de lâmina d'água, em função da aplicação pós-emergente do herbicida triclopir-butotílico nas doses 0.0x (zero); 0.5x (166 g. ia. ha-1); 1x (336 g. ia. ha-1); 2x (672 g. ia. ha-1); 4x (1.344 g. ia. ha-1); 8x (2.688 g. ia. ha-1) e 16x (5.376 g. ia. ha-1) de registro. Santa Maria/RS, 2017.

Os resultados observados neste estudo permitem inferir que o cultivo contínuo de arroz CL e com altas doses de herbicidas associado à baixa rotação de culturas, favorece a alta pressão de seleção de biótipos de Cyperus. A resistência ocorre principalmente a herbicidas bispiribaquesódio, imazapic, imazethapyr, penoxsulam e pirazosulfuronetil, envolvendo mais de um grupo químico do mecanismo de ação (HEAP, 2018). Assim, deve adotar alternativas de manejo dos cultivos, a fim de controlar as plantas daninhas, reduzindo as perdas de produtividade e evitando a dispersão das mesmas pelas áreas de produção.

\section{Conclusões}

O biótipo $C$. difformis de Meleiro-RS é $8 \mathrm{x}$ (320 g. i.a. ha $^{-1}$ ) mais resistente a dose de registro do bispiribaque-sódico. Já o biótipo de Santa Rosa do Sul-SC é mais suscetível, reduzindo $50 \%$ da massa seca da parte aérea com $0.5 \mathrm{x}$ da dose de registro ( $20 \mathrm{~g}$ i.a. $\left.\mathrm{ha}^{-1}\right)$.

O pirazossulfurom-etílico, nas doses testadas, demostrou-se ineficiente na redução da massa seca da parte aérea para os biótipos de C. difformis de Santa Rosa do SulSC e Meleiro-SC.

Os biótipo C. difformis de Santa Rosa do Sul-SC e Meleiro-SC, nas duas condições de umidade do solo, são suscetíveis ao triclopir-butotílico, com redução de $50 \%$ da massa seca da parte aérea com $0.5 x\left(166\right.$ g. i.a. ha $\left.{ }^{-1}\right)$ da dose de registro.

\section{Referências}

Agostinetto, D. et al. Resistência de Cyperus difformis L. ao herbicida pyrazosulfuron-ethyl e alternativas de controle.
Semina: Ciências Agrárias, v. 32, n. 3, p. 839- 848, 2011.

Bonny, S. Herbicide-tolerant Transgenic Soybean over 15 Years of Cultivation: Pesticide Use, Weed Resistance, and Some Economic Issues. The Case of the USA. Sustainability 3 (5): 1302-1322, 2011.

Chiapinotto, D. M., Schaedler, C. E., Fernandes, J. P. S., Andres, A., Lamego, F. P. Cross-resistance of rice flatsedge to als-inhibiting herbicides. Planta Daninha, 35. p. e017166827, 2017.

Christoffoleti, PJ.; López-ovejero, R.E. Resistência das plantas daninhas a herbicidas: definições, bases e situação no Brasil e no mundo. ln: Christoffoleti, P.J. (Coord.). Aspectos de resistência de plantas daninhas a herbicidas. 3.ed. Piracicaba: Associação Brasileira de Ação a Resistência de Plantas aos Herbicidas - HRAC-BR, 2008. p. 9-34

CONAB - Companhia Nacional de Abastecimento. Acompanhamento da Safra Brasileira de Grãos, 2019. Disponível em: <http://www.conab.gov.br/conteudos.php? $\mathrm{a}=1253 \&>$. Acesso em: 12 jan. 2020.

Galon, L.; Panozzo, L.E.; Noldin, J.A.; Concenço, G.; Tarouco, C.P.; Ferreira, E.A.; Agostinetto, D.; Silva, A.A.; Ferreira, F.A. Resistência de Cyperus difformis a herbicidas inibidores da ALS em lavoura de arroz irrigado em Santa Catarina. Planta Daninha, Viçosa-MG, v. 26, n. 2, p. 419427, 2008.

Heap, I. The International Survey of Herbicide Resistant Weeds. 2009. <Disponível em: http:// www.weedscience.org > . Acesso em: 8 maio 2019. 
Heap, I. The International Survey of Herbicide Resistant weeds. Online. Internet. Saturday, January 28, 2017.

Heap I. Levantamento internacional de ervas daninhas resistentes a herbicidas. 2018. Disponível em: Disponível em: <http://www.weedscience.org $>$. Acesso em: 8 maio 2019.

Hofstra, D. E.; Champior, P. D., Dudgale, T. M. Herbicide trials for the control of parrotsfeather. J. Aquati. Plant Management. v. 44, p. 13-18, 2006.

Noldin, J. A.; Eberhardt, D. S.; Rampelotti, F. T. Cyperus difformis L. resistente a herbicidas inibidores da ALS em Santa Catarina. In: CONGRESSO BRASILEIRO DA CIÊNCIA DAS PLANTAS DANINHAS, 2, Gramado, 2002. Resumos... Londrina: SBCPD/Embrapa Clima Temperado, p. 198, 2002.

Osuna, M. D.; Vidotto, F.; Fischer, A. J.; Bayer, D. E.; Prado, R. D.; Ferrero, A. Cross-resistance to bispyribacsodium and bensulfuron-methyl in Echinochloa phyllopogon and Cyperus difformis. Pesticide Biochemistry \& Physiology, v. 73, n. 1, p. 9-17, 2002.

Pacanoski, Z.; Glatkova, G. The Use of Herbicides for Weed Control in Direct Wet-Seeded Rice (Oryza sativa L.) in Rice Production Regions in the Republic of Macedonia. Plant Protection Science, v. 45, n. 3, p. 113-118, 2009.

Pedroso, R. M., Neto, D. D., Victoria Filho, R., Fischer, A. J., Al-Khatib, K. Modeling germination of smallflower umbrella sedge (Cyperus difformis L.) seeds from rice fields in California across suboptimal temperatures. Weed Technology, v. 33, n. 5, p. 733-738, 2019.

SBCPD - Sociedade Brasileira da Ciências das Plantas Daninhas. Procedimentos para instalação, avaliação e análise de experimentos com herbicidas. Londrina: SBCPD, p. 41, 1995.

Seefeldt, S. S.; Jensen, J. E., Fuerst, E. P. Log-logistic analysis of herbicide dose-response relationships. Weed technology 9 (2): p. 218-227, 1995.

SOSBAI - Sociedade Sul-Brasileira de Arroz Irrigado. Arroz irrigado: recomendações técnicas da pesquisa para o Sul do Brasil. XXX Reunião Técnica da Cultura do Arroz Irrigado. Bento Gonçalves - RS, Brasil. p. 35, 2014.

SOSBAI - Sociedade Sul-Brasileira de Arroz Irrigado. Arroz irrigado: Recomendações técnicas da pesquisa para o Sul do Brasil. Santa Maria: Universidade Federal de Santa Maria, p. 9, 2018.

Spatt, L. L. et al. Manejo de junquinho com aplicação isolada e sequencial de diferentes herbicidas em arroz irrigado. Anais do XI Congresso Brasileiro de Arroz Irrigado - CBAI. Pelotas, 2015.
Schwab, N. T. Disponibilidade hídrica no cultivo de cravina em vasos com substrato de cinzas de casca de arroz. 2011. 82 f. Dissertação (Mestrado em Engenharia Agrícola: Área de Concentração Engenharia de água e solo) - Universidade Federal de Santa Maria, Santa Maria, 2011.

Tembe, Á. L. B. Avaliação do efeito do método físico, químico e mecânico no controlo da tiririca (Cyperus rotunds L.) na cultura do milho (Zea mays L.). 2014. Dissertação de Mestrado. Universidade Eduardo Mondlane.

Tian, Z., Shen, G., Yuan, G., Song, K., Lu, J., Da, L. Effects of Echinochloa crusgalli and Cyperus difformis on yield and eco-economic thresholds of rice. Journal of Cleaner Production, p. 120807, 2020.

Ulguim, A. R. et al. Resistance mapping of the genus Cyperus in Rio Grande do Sul and selection Pressure analysis. Planta Daninha, Viçosa, v. 37, 2019. 